\title{
Modeling of the spectrum in a random distributed feedback fiber laser within the power balance modes
}

\author{
Ilya D. Vatnik ${ }^{\mathrm{a}, *}$, Dmitry V. Churkin ${ }^{\mathrm{a}, \mathrm{b}, \mathrm{c}}$ \\ anstitute of Automation and Electrometry SB RAS, ac. Koptyug ave. 1, Novosibirsk, 63009, Russia; \\ ${ }^{\mathrm{b}}$ Aston Institute of Photonic Technologies, Aston University, Birmingham, B4 7ET, United \\ Kingdom; 'Novosibirsk State University, Pirogova str. 2, Novosibirsk 630090, Russia
}

\begin{abstract}
The simplest model for a description of the random distributed feedback (RDFB) Raman fiber laser is a power balance model describing the evolution of the intensities of the waves over the fiber length. The model predicts well the power performances of the RDFB fiber laser including the generation threshold, the output power and pump and generation wave intensity distributions along the fiber. In the present work, we extend the power balance model and modify equations in such a way that they describe now frequency dependent spectral power density instead of integral over the spectrum intensities. We calculate the generation spectrum by using the depleted pump wave longitudinal distribution derived from the conventional power balance model. We found the spectral balance model to be sufficient to account for the spectral narrowing in the RDFB laser above the threshold of the generation.
\end{abstract}

Keywords: Random laser, Raman laser, Rayleigh backscattering, balance model

\section{INTRODUCTION}

The concept of the irregular feedback being exploited for lasing was introduced by Letokhov in 1960s, initially in the context of study of microwave generation in the giant interstellar gas and dust clouds ${ }^{1}$, and was experimentally demonstrated by Ambartsumyan and others ${ }^{2}$ substituting a diffusor for one of mirrors of a Ruby laser. In such a system the generated light doesn't accomplish exact isolated round-trips, as reflecting from different parts of bulk diffusor. In other words, there are a lot of low-finesse modes highly overlapped, so light energy may easily flow from one mode to another. Therefore, the system with scatterers playing role of a mirror has none of the isolated modes present in the conventional Fabry-Perot cavity, and can be treated, following Ambartsumyan ${ }^{2}$, as having one effective mode spreading over whole amplification spectral region. Hence, spectral properties should be determined by the amplification properties of the media rather than by resonator particular structure. Such a feedback has been named nonresonant.

After the mentioned works, the idea of irregular feedback had originated new field of random lasers, which comprises scatterers randomly distributed in active media. Despite the lack of the conventional cavity, the random active systems have typical for lasers features such as threshold dependence of output power on pump rate and dramatic spectral narrowing above the threshold. Random lasing has been demonstrated in a variety of different active media, including active powders ${ }^{3},{ }^{4}$, dye solutions with nanoparticles suspended ${ }^{5}$, polymer films doped with both dye and particles ${ }^{6}$, and many others ${ }^{7,8}$. It was shown $\mathrm{in}^{3,4}$ that in the case of strong scattering despite the irregular nature of the feedback some modes arising due to interference of multiply scattered photons can retain and generation occur only in these modes. Such regime was referred to as resonant feedback opposite to nonresonant regime discussed above. There can be a transition from resonant to nonresonant regime in one system. If the system is excited by the pump beam of a small beam spot, few modes only are excited resulting in resonant random feedback lasing with fine structure of several narrow lines, corresponding to different retaining modes. Changing the shape of pump spot and increasing its size leads to growth of a number of modes involved in generation. When it is sufficiently large, different randomly located modes overlap and the smooth spectrum corresponding to the non-resonant feedback is generated ${ }^{9}$.

*ilya.vatnik@gmail.com

Laser Sources and Applications II, edited by Jacob I. Mackenzie, Helena Jelĺnková, Takunori Taira, Marwan Abdou Ahmed, Proc. of SPIE Vol. 9135, 91351Z · (C) 2014 SPIE · CCC code: 0277-786X/14/\$18 · doi: 10.1117/12.2052328 
Recently, a different type of a random laser-random distributed feedback (RDFB) fiber laser based on standard optical fiber with irregular feedback by Rayleigh backscattering is proposed and demonstrated ${ }^{10}$. Possessing some distinguishing features such as quasi-CW generation with directional output, the laser demonstrates processes of spectral narrowing over the gain bandwidth at the threshold to the smooth spectrum without fine structure. This together with fact of extreme weakness of Rayleigh backscattering process could indicate the non-coherent type of the feedback realized in the RDFB fiber laser.

Up to date, a number of different schemes of random DFB fiber lasers were demonstrated ${ }^{11-26}$. Namely, random DFB fiber lasers can operate in different spectral bands ${ }^{12,13}$, emit higher order Stokes waves ${ }^{12-14}$, be tunable ${ }^{22,23}$ and multiwavelength ${ }^{15,19,21}$. The noise level of random DFB fiber lasers could be lower than of conventional lasers ${ }^{27}$ making them attractive for telecom applications. Random DFB fiber lasers are also applied for sensor applications $\mathrm{s}^{28-30}$.

The modelling of the random DFB fiber lasers is of interest, however only few works devoted to this. The simplest model is the power balance model ${ }^{10,26}$ which describes well the average power performances such as the generation threshold $^{10}$, the output power value, the longitudinal distribution of the power ${ }^{26}$ and can be used to perform a laser optimization $^{31}$. To deal with spectral and temporal properties of the laser radiation, NLSE-based model usually used ${ }^{32}$. They are implemented for the conventional lasers such as Brillouin lasers ${ }^{33}$, Ytterbium-doped fiber lasers ${ }^{34,35}$ and Raman fiber lasers (RFLs) ${ }^{36-39}$. Recently, the NLSE-based model has been also applied for the description of the random DFB fiber laser ${ }^{40}$. The advantage of this model is the complete description of the generation properties in both spectral and time domain.

However, the balance model is much easier to work with and demands considerably lower computation capabilities. So it is of interest to describe the spectral properties of the random DFB fiber laser within the power balance model. Here we suggest the spectral power balance model for the RDFB Raman fiber laser with feedback based on Rayleigh backscattering. The model operates with spectral power densities of generation. Rayleigh backscattering is considered as nonresonant feedback with no interference effects. We perform numerical simulation of the spectra near and at the threshold of generation, using a simple approximation of the given pump distribution.

\section{SPECTRAL BALANCE MODEL}

To calculate spectral shape of the generation within balance approach, we have changed well-known power balance equation set describing power transfer from the pump to the Stokes waves ${ }^{10,26,41}$. This model takes into consideration power income to the Stokes wave due to Raman amplification, outgo from the pump wave known as depletion, linear losses every optical fiber possesses, and Rayleigh backscattering process which mixes Stokes waves propagating in different directions:

$$
\left\{\begin{array}{l}
\left(\alpha_{P} \pm d / d z\right) P_{p}=-g_{\max } P_{p}\left(P_{S}^{+}+P_{S}^{-}+4 h v_{S} \Delta v\right) \frac{v_{P}}{v_{S}} \\
\left(\alpha_{S} \pm d / d z\right) P_{S}^{ \pm}=g_{\max } P_{p}\left(P_{S}^{ \pm}+2 h v_{S} \Delta v\right)+\varepsilon_{S} \cdot P_{S}^{\mp}
\end{array} .\right.
$$

Here P denotes the power, lower indexes $P, S$ refer the corresponding terms to pump and Stokes waves, respectively, while upper indexes + and - refer to forward (propagating in the direction along the $z$ axis) and backward (corresponding to $z$ axis) propagating waves, respectively. Coefficient $\alpha$ defines the linear losses, $g_{\max }$ is the maximum Raman gain coefficient at the peak of amplification spectrum, $v$ is the radiation frequency, $\Delta v$ is the Raman amplification spectrum bandwidth, $\varepsilon$ is the backscattering coefficient, derived as $\alpha_{s}$ multiplied by backscattering factor $Q$. Backscattered factor is determined by geometrical measures of the fiber and is the part of scattered radiation which is then recaptured by the fiber waveguide and starts to propagate in opposite direction.

It was proved that power balance model describes well distributions of the generated power ${ }^{26,31}$. Note that in the model (1) the generation wave is assumed to be a single-frequency radiation having a wavelength corresponding to the maximum Raman gain. This simplification gives the adequate results due to rather narrow spectrum of the RDFB lasers (of 1 nanometer) compared with the spectral bandwidth of the Raman amplification (tens of nanometers).

Here we make a further step and introduce a spectral balance mode by assuming that the pump wave is depleted by number of Stokes wave generated at different frequencies within the Raman gain profile. In other words, we use spectral power densities $P_{s}(\lambda)$ instead of the total powers $P_{s}$. 


$$
\left\{\begin{array}{c}
\left(\alpha_{P} \pm d / d z\right) P_{p}=-g_{\max } P_{p}\left(\int g(\lambda) P_{s}^{+}(\lambda) d \lambda+\int g(\lambda) P_{s}^{-}(\lambda) d \lambda+4 h v_{S} \Delta v\right) \frac{v_{P}}{v_{S}} \\
\left(\alpha_{S} \pm d / d z\right) P_{S}^{ \pm}(\lambda)=g(\lambda) P_{p}\left(P_{S}^{ \pm}(\lambda)+2 h v_{S} \Delta v\right)+\varepsilon_{S} \cdot P_{S}^{\mp}(\lambda)
\end{array}\right.
$$

Here we use $P_{S}(\lambda)$ to denote spectral power density of the Stokes wave. Note that the equation (3) governing the evolution of the Stokes wave $P_{S}(\lambda)$ comprises the wavelength dependent Raman gain coefficient $g(\lambda)$ instead of constant over wavelength Raman gain in power balance model (1).

The power balance model does not comprise Kerr nonlinear coefficient, so it can not describe nonlinear effects such as four-wave-mixing inside the Stokes wave spectrum or cross-phase modulation between the pump and the Stokes waves. Meanwhile, it is well known that nonlinear broadening is a crucial factor determining spectral shape of the generation of Raman fiber lasers ${ }^{43-45}$ and the spectral width of the random DFB fiber lasers depends both on nonlinearity and dispersion $^{40}$. We consider here only the region of the pump power under and near the generation threshold, where nonlinear effect are negligible because of small Stokes wave power.

We find the generation Stokes wave spectrum by finding the generation power $P_{S}(\lambda)$ from Eq.(3) at each fixed wavelength. To do that one needs to know the distribution of the pump power over the fiber $P_{P}(z)$. To find the pump power distribution, we use the power balance model (1) as it provides a quantitative description of the pump power distribution ${ }^{26,31}$. The obtained pump power distribution $P_{P}(z)$ is then used in equations (3) to find the spectral shape of $P_{S}(\lambda)$. In this case equations (3) at different wavelengths can be separated, and the pairs of equations on $P_{s}^{+}(\lambda)$ and $P_{s}^{-}(\lambda)$ for each wavelength are solved independently.

As we have already mentioned above, the balance model (1) proved to describe well the power distributions of the Stokes wave in the RDFB laser, thus use of pump power distribution $P_{P}(z)$ found from (1) is reliable.

Further we consider the one-arm configuration of the random DFB fiber laser, Fig. 1. In this scheme tpe pump power is launched into the fiber span from one side. So the boundary conditions for the laser read

$$
\left\{\begin{array}{c}
P_{p}(0)=P_{i n} \\
P_{s}^{+}(0)=P_{s}^{-}(L)=0
\end{array}\right.
$$

where $P_{\text {in }}$ is input pump power and $L$ is fiber length. To solve the boundary problem $(3,4)$ the shooting method is used.

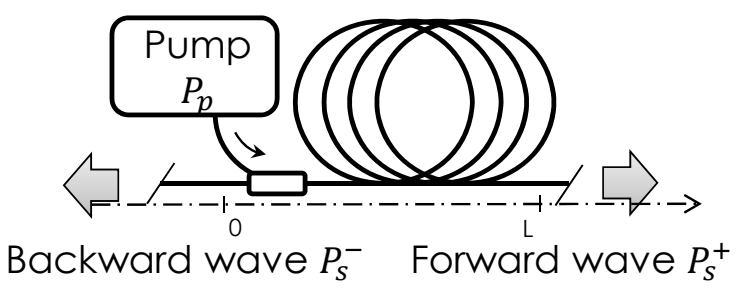

Figure 1. Setup under investigation

We suggest the $1455 \mathrm{~nm}$ pump creating amplification at around $1550 \mathrm{~nm}$. As a test-bed of the approach, parameters for the typical commercially available nonzero-dispersion-shifted fiber are used for simulation. The parameters were estimated from the manufacturer data or directly measured and are as follows: $\alpha_{s}=0.051 / \mathrm{km}, \alpha_{p}=0.041 / \mathrm{km}, Q=0.0007$, $\mathrm{L}=50 \mathrm{~km}, g_{R}=0.81 / \mathrm{W} / \mathrm{km}$. We use the spectral shape of the Raman gain in conventional single mode fibers taken from ${ }^{42}$.

\section{RESULTS AND DISCUSSION}

As was found from the integral balance model (1), the threshold power for the system under investigation is around 0.8 W. On the fig.2 the pump distributions $P_{P}(z)$ derived from (1) are depicted for two different input pump powers. While 
under the threshold $(0.7 \mathrm{~W})$ the pump distribution is well described by the exponential decay function $P_{P}(z) \sim \exp \left(-\alpha_{\mathrm{p}} \mathrm{z}\right)$. While above the threshold $(0.9 \mathrm{~W})$ pump wave attenuates faster because of energy transfer into Stokes wave.

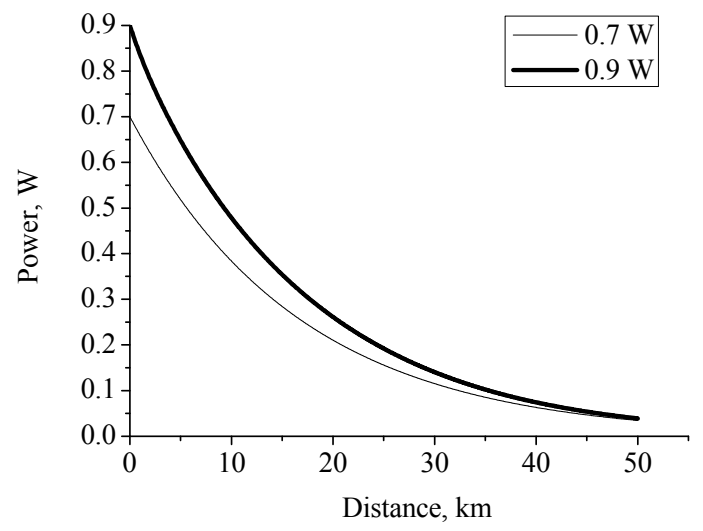

Figure 2. Pump distributions $P_{P}(z)$.

The results of the spectral modeling for different pump powers are shown on Fig.3(a). For the small pump powers, only broadband spectrum corresponding to amplified spontaneous emission is generated. With the pump power increase, the spectrum of ASE gradually narrows, demonstrating the "superluminescent" regime well known for different types of lasers. But after a certain pump power level, the output spectrum narrows abruptly, Fig.3(b). As the same time, total generated Stokes power found as $\int P_{s}^{-}(\lambda) d \lambda$ starts to grow up. Thus, this is a generation threshold. We found from our simulations the threshold pump power value of $0.8 \mathrm{~W}$ that is well coincides with previously experimentally demonstrated threshold for the similar laser schemes ${ }^{10}$. Above the threshold, the generation spectrum has a form of a distinct wellpronounced single peak situated at the maximum of Raman amplification profile.

(a)

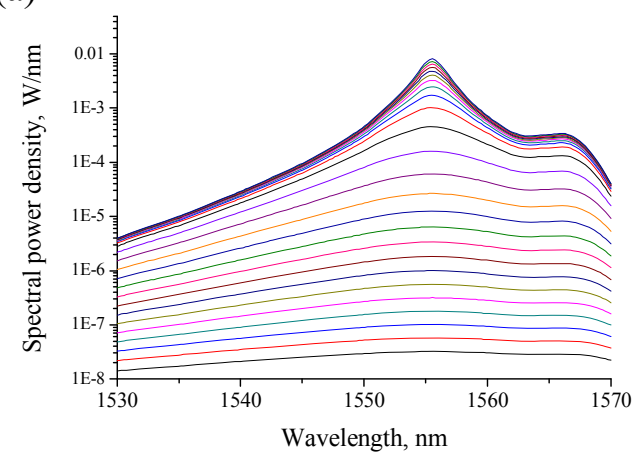

(b)

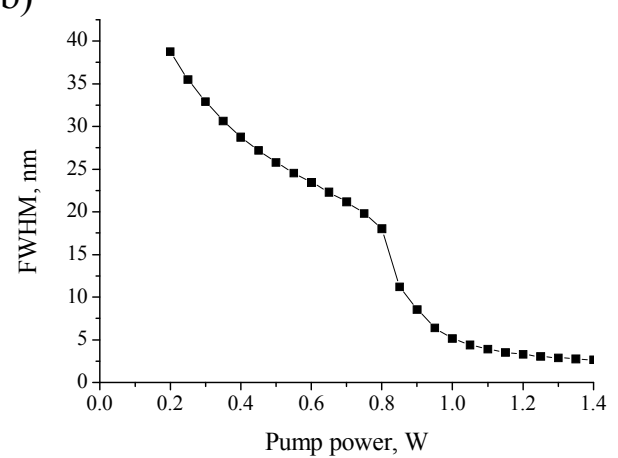

Figure 3. (a) Numerically obtained generation spectra of the random DFB fiber laser under different pumping. The pump power increases from 0.2 to $1.4 \mathrm{~W}$ with $0.05 \mathrm{~W}$ step. (b) The full width at half maximum of the Stokes wave spectrum

Integrating the generation spectrum over the wavelength, one can find the total generation power, Fig. 4. In our case, the total Stokes power calculated above the threshold is too small compared to previous experimental data: in experiments slope efficiency of more than $60 \%$ is reported ${ }^{31}$ in one-arm schemes while it's of $5 \%$ in the simulation. This means that despite the generation spectrum has a spectrum shape in qualitative agreement with experiments, further studies should be undertaken to achieve a quantitative agreement. In particular, the pump power distribution should be accounted more accurately by taking into account the pump power depletion governed by Eq.(2) instead of Eq. (1). 


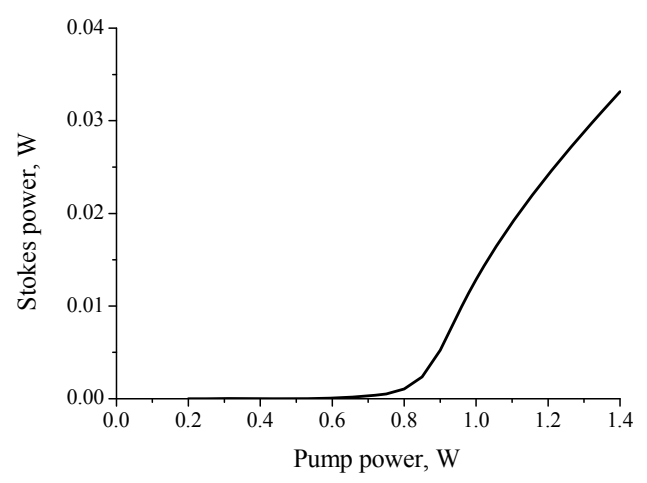

Figure 3. Output generation power obtained by integration the spectra simulated.

\section{CONCLUSION}

To conclude, we suggest a simple approach to account for an impact of Rayleigh backscattering on spectral properties of the random distributed feedback fiber laser. We extend the power balance model and modify equations in such a way that they describe now frequency dependent spectral power density instead of integral over the spectrum intensities. We implement the approach of the given pump distribution, which is derived from power balance model avoiding spectral shape of the radiation and then is used to find spectral power densities of the generation. The model describes in the qualitative way the generation spectrum of the random DFB fiber laser. In particular, one can trace how the spectrum acquires its final spectral shape starting from initial broad gain spectrum.

\section{REFERENCES}

[1] Letokhov, V. S. "Stimulated radio emission of the interstellar medium," Lett. to J. Exp. Theor. Phys. 4(11), 321 (1966).

[2] Ambartsumyan, R. V, Basov, N. G., Kryukov, P. G., and Letokhov, V. S. "A laser with nonresonant feedback," J. Experimantal Theor. Phys. 24(3), 481-485 (1967).

[3] Cao, H., Zhao, Y. G., Ong, H. C., Ho, S. T., Dai, J. Y., Wu, J. Y., and Chang, R. P. H. "Ultraviolet lasing in resonators formed by scattering in semiconductor polycrystalline films," Appl. Phys. Lett. 73(25), 3656 (1998).

[4] Cao, H. , Zhao, Y., Ho, S., Seelig, E., Wang, Q., and Chang, R. "Random laser action in semiconductor powder," Phys. Rev. Lett. 82(11), 2278-2281 (1999).

[5] Lawandy, N. M. , Balachandran, R. M., Gomes, A. S. L., and Sauvain, E. "Laser action in strongly scattering media," Nature 368(6470), 436-438 (1994).

[6] Zacharakis, G., Papadogiannis, N. A., Filippidis, G., and Papazoglou, T. G., "Photon statistics of laserlike emission from polymeric scattering gain media," Opt. Lett. 25(12), 923 (2000)

[7] Wiersma, D. S. "The physics and applications of random lasers," Nat. Phys. 4(5), 359-367 (2008)

[8] Cao, H. "Review on latest developments in random lasers with coherent feedback," J. Phys. A. Math. Gen. 38(49), 10497-10535 (2005).

[9] Leonetti, M., Conti, M., and Lopez, C. "The mode-locking transition of random lasers," Nat. Photonics 5(10), 615-617 (2011).

[10] Turitsyn, S. K., Babin, S.A., El-Taher, A. E., Harper, P., Churkin, D. V., Kablukov, S. I., Ania-Castañón, J. D. , Karalekas, V., and Podivilov, E. V., "Random distributed feedback fibre laser," Nat. Photon. 4, 231-235 (2010).

[11] Churkin, D. V., Babin, S. A., El-Taher, A. E. , Harper, P., Kablukov, S. I., Karalekas, V., Ania-Castañón, J. D., Podivilov, E.V., and Turitsyn, S. K., "Raman fiber lasers with a random distributed feedback based on Rayleigh scattering," Phys. Rev. A 82, 033828 (2010).

[12] Vatnik, I. D., Churkin, D. V., Babin, S. A., and Turitsyn, S. K. "Cascaded random distributed feedback Raman fiber laser operating at $1.2 \mu \mathrm{m}$," Opt. Express 19, 18486-18494 (2011). 
[13] Teng, R. , Ding, Y., and Chen, L. "Random fiber laser operating at 1,115 nm," Appl. Phys. B, 111, 169-172, (2013).

[14] Zhang, W. L., Rao, Y. J., Zhu, J. M., Yang, Z. X., Wang, Z. N., and Jia, X. H. "Low threshold 2nd-order random lasing of a fiber laser with a half-opened cavity," Opt. Express 20, 14400-5 (2012).

[15] Pinto, A. M. R., Frazão, O., Santos, J. L., and Lopez-Amo, M. "Multiwavelength fiber laser based on a photonic crystal fiber loop mirror with cooperative Rayleigh scattering," Appl. Phys. B 99, 391-395 (2010).

[16] Zhang, W. L., Zhu, Y. Y., Rao, Y. J., Wang, Z. N., Jia, X. H., and Wu, H. "Random fiber laser formed by mixing dispersion compensated fiber and single mode fiber," Opt. Express 21, 8544 (2013).

[17] Sarmani, A. R., Abu Bakar, M. H. , Bakar, A. A. A., Adikan, F. R. M., and Mahdi, M. A. "Spectral variations of the output spectrum in a random distributed feedback Raman fiber laser," Opt. Express 19, 14152-14159 (2011).

[18] Sarmani, A. R., Abu Bakar, M. H., Adikan, F. R. M., and Mahdi, M. A. "Laser parameter variations in a rayleigh scattering-based raman fiber laser with single fiber bragg grating reflector." IEEE Photonics Journal 4, 461-466 (2012).

[19] El-Taher, A. E., Harper, P., Babin, S. A., Churkin, D.V., Podivilov, E. V, Ania-Castañón, J. D., and Turitsyn, S. K., "Effect of Rayleigh-scattering distributed feedback on multiwavelength Raman fiber laser generation," Opt. Lett. 36, 130-132 (2011).

[20] Sugavanam, S., Tarasov, N., Shu, X., and Churkin, D. V. "Narrow-band generation in random distributed feedback fiber laser," Opt. Express 21(14), 16466-16472 (2013).

[21] Sugavanam, S., Yan, Z., Kamynin, V., Kurkov, A. S., Zhang, L. and Churkin, D. V. "Multiwavelength generation in a random distributed feedback fiber laser using an all fiber Lyot filter," Opt. Express 22, 28392844 (2014).

[22] Sarmani, A., Zamiri, R., and Bakar, M. "Tunable Raman fiber laser induced by Rayleigh backscattering in an ultra-long cavity," Journal of the European Optical society, 6, 11043 (2011).

[23] Babin, S. A. , El-Taher, A. E., Harper, P., Podivilov, E. V., and Turitsyn, S. K. "Tunable random fiber laser," Phys. Rev. A 84, 021805 (2011).

[24] Pang, M., Xie, S., Bao, X., Zhou, D., Lu, Y., and Chen, L. "Rayleigh scattering-assisted narrow linewidth Brillouin lasing in cascaded fiber," Opt. Lett.37, 3129-3131 (2012).

[25] Rao, Y. J., Zhang, L. W., Zhu, J. M., Yang, Z. X., Wang, Z. N., and Jia, X. H. "Hybrid lasing in an ultra-long ring fiber laser," Opt. Express 20, 22563-22568 (2012).

[26] Churkin, D. V., El-Taher, Vatnik, I. D., Ania-Castañón, J. D., Harper, P., Podivilov, E. V., Babin, S. A. and Turitsyn, S. K. "Experimental and theoretical study of longitudinal power distribution in a random DFB fiber laser," Opt. Express 20, 11178-11188 (2012).

[27] Nuño, J., Alcon-Camas, M., and Ania-Castanon, J. D. "RIN transfer in random distributed feedback fiber lasers," Opt. Express 20, 27376-27381 (2012).

[28] Pinto, A. M. R., Lopez-Amo, M.. Kobelke, J., and Schuster, K. "Temperature fiber laser sensor based on a hybrid cavity and a random mirror," J. Lightwave Technol. 30, 1168-1172 (2012).

[29] Pinto, A. M. R., Frazão, O., Santos, J. L., Lopez-Amo, M.., Kobelke, J. and Schuster, K. "Interrogation of a suspended-core Fabry-Perot temperature sensor through a dual wavelength Raman fiber laser," J. Lightwave Technol. 28, 3149-3155 (2010).

[30] Martins, H. F., Marques, M. B, and Frazão, O. "Temperature-insensitive strain sensor based on four-wave mixing using Raman fiber Bragg grating laser sensor with cooperative Rayleigh scattering," Appl. Phys. B 104, 957-960 (2011).

[31] Vatnik, I. D. , Churkin, D. V., and Babin, S. A. "Power optimization of random distributed feedback fiber lasers," Opt. Express 20, 28033-28038 (2012)

[32] Agrawal, G. P., "Nonlinear fiber optics," Academic Press (2001).

[33] Preda, C. E., Ravet, G., Fotiadi, A. A., and Mégret, P. "Iterative method for Brillouin fiber ring resonator," in CLEO/Europe 2011 Conference, OSA Technical Digest (Optical Society of America, 2011), paper CJ_P27.

[34] Turitsyn, S. K., Bednyakova, A. E., Fedoruk, M. P., Latkin, A. I., Fotiadi, A. A., Kurkov, A. S., and Sholokhov, E. "Modeling of CW Yb-doped fiber lasers with highly nonlinear cavity dynamics," Opt. Express 19, 1227-1230 (2011).

[35] Bednyakova, A. E., Gorbunov, O.A., Politko, M. O., Kablukov, S. I., Smirnov, S. V., Churkin, D. V., Fedoruk, M. P. and Babin, S. A. "Generation dynamics of the narrowband Yb-doped fiber laser," Opt. Express 21, 8177$8182(2013)$. 
[36] Churkin, D. V., Smirnov, S. V., and Podivilov, E. V. "Statistical properties of partially coherent CW fiber lasers," Opt. Lett. 35, 3288-3290 (2010).

[37] Randoux, S., Dalloz, N., and Suret, P. "Intracavity changes in the field statistics of Raman fiber lasers," Opt. Lett. 36, 790-792 (2011).

[38] Churkin, D. V., Gorbunov, O. A., and Smirnov, S. V. "Extreme value statistics in Raman fiber lasers," Opt. Lett. 36, 3617-3619 (2011).

[39] Churkin, D. V., and Smirnov, S. V. "Numerical modelling of spectral, temporal and statistical properties of Raman fiber lasers," Opt. Commun. 285, 2154-2160 (2012).

[40] Smirnov, S. V. and Churkin, D. V. "Modeling of spectral and statistical properties of a random distributed feedback fiber laser." Opt. Express, 21, 21236-21241 (2013).

[41] Smith, R. G. "Optical Power Handling Capacity of Low Loss Optical Fibers as Determined by Stimulated Raman and Brillouin Scattering," Appl. Opt. 11(11), 2489-2494 (1972).

[42] Lin Q., and Agrawal, G. P. "Raman response function for silica fibers.," Opt. Lett. 31(21), 3086-3088 (2006).

[43] Babin, S. A., Churkin, D. V., Ismagulov, A. E., Kablukov, S. I., and Podivilov, E. V. "FWM-induced turbulent spectral broadening in a long Raman fibre laser," J. Opt. Soc. Am. B 24, 1729-1738 (2007).

[44] Babin, S. A., Churkin, D. V., Ismagulov, A. E., Kablukov, S. I., and Podivilov, E. V., "Spectral broadening in Raman fiber lasers," Opt. Lett. 31, 3007-3009 (2006).

[45] Babin, S. A., Churkin, D. V., Ismagulov, A. E., Kablukov, S. I., and Podivilov, E. V. "Turbulence-induced square-root broadening of the Raman fiber laser output spectrum," Opt. Lett. 33, 633-635 (2008). 